\title{
Sufficiency Conditions for Finite Escape Times in Systems of Quadratic Differential Equations
}

\author{
W. M. GetZ AND D. H. JAcobson \\ National Research Institute for Mathematical Sciences, CSIR, \\ P.O. Box 395, Pretoria 0001, Republic of South Africa
}

[Received 12 June 1975 and in revised form 20 April 1976]

Sufficient conditions are derived for solutions of a general autonomous system of quadratic ordinary vector differential equations to exhibit finite escape times. These results are extended to a matrix Riccati-type differential equation using Kronecker matrix products and a "stacking operator". Finally, these results are applied to a class of models used extensively in population dynamics.

\section{Introduction}

GENERAL procedures are well-known for analysing the stability properties of systems of non-linear ordinary differential equations. Important among these procedures are:

(i) the various Lyapunov-type theorems (Hahn, 1967; Cesari, 1963, chapter 3) and

(ii) the analysis of the stability properties of the corresponding variational system, viz. the non-linear system linearized about one of its stationary points (Willems, 1970, chapter 5).

Stability results pertaining to specific systems are known for many different types of non-linear differential equations. Often these results are derived by finding a suitable Lyapunov function for the system concerned and accordingly the applicability of the results is limited to that system.

However, few explicit results exist for the stability properties of quadratic differential equations, especially since suitable Lyapunov functions for general quadratic systems are difficult to find. The primary reason for this is that the time derivative of a scalar function of even order is odd and it is therefore difficult to comment upon its sign without knowing more about the structure of the solutions of the quadratic differential equations.

In this paper we derive sufficiency conditions for solutions of autonomous systems of quadratic differential equations to have a finite escape time. An upper bound for the escape time is also given.

Using the Kronecker matrix product and an operator which stacks columns of a matrix into an extended vector, we show that the matrix Riccati-equation that arises in the linear-quadratic regulator problem of optimal control theory, can be rewritten into a format such that our results complement known sufficient conditions of the Kalman-type (Jacobson, 1970).

Finally, we demonstrate that the results derived in this paper can be applied to a general class of systems of autonomous quadratic differential equations used to model interacting populations. The Lotka-Volterra model of predator-prey dynamics is a special case of this class. 


\section{Sufficiency Result}

In this section we derive sufficient conditions for the solution to the quadratic system

$$
\dot{x}_{i}=\mathbf{x}^{T} A_{i} \mathbf{x}+\mathbf{b}_{i}^{T} \mathbf{x}+c_{i} \quad i=1, \ldots, n \quad \mathbf{x}\left(t_{0}\right)=\mathbf{x}_{0}
$$

to escape in a finite time, i.e. $\left\|\mathbf{x}\left(t ; \mathbf{x}_{0}, t_{0}\right)\right\| \rightarrow \infty$ as $t \rightarrow t_{e}<\infty$. In (1) the $n \times n$ matrices $A_{i}$ are constant and without loss of generality can be assumed to be symmetric. The $n$-vectors $\mathbf{b}_{i}$ and the scalars $c_{i}$ are constant.

To facilitate a compact notation the following definitions are made. For any constant vector $\omega \in \mathbb{R}^{n}$ with $i$ th element $\omega_{i}$, define

$$
\begin{aligned}
A_{\omega} & =\sum_{i=1}^{n} \omega_{i} A_{i}, & \mathbf{b}_{\omega} & =\sum_{i=1}^{n} \omega_{i} \mathbf{b}_{i}, \\
c_{\omega} & =\sum_{i=1}^{n} \omega_{i} c_{i}, & \Delta_{\omega} & =\left(\lambda_{\omega} / \omega^{T} \omega\right)\left(\mathbf{b}_{\omega}^{T} A_{\omega}^{-1} \mathbf{b}_{\omega}-4 c_{\omega}\right),
\end{aligned}
$$

where $\lambda_{\omega}$ is the smallest eigenvalue of $A_{\omega}$. It is worth noting that $\lambda_{\omega}$ is real since each $A_{i}$ was assumed to be symmetric. Further $\lambda_{\omega}>0$ if $A_{\omega}>0$.

Theorem 2.1. Consider the system of equations given in (1). Suppose there exists a vector $\omega \in \mathbb{R}^{n}$ such that $A_{\omega}>0$ (positive definite). Then the solution of system (1) has a finite escape time $t_{e}$ under the following conditions:

(i) for all $\mathbf{x}_{0}$, whenever $\Delta_{\omega}<0$;

(ii) for $\mathbf{x}_{0}$ satisfying

whenever $\Delta_{\omega} \geqslant 0$.

$$
\omega^{T} \mathbf{x}_{0}>-\frac{1}{2} \omega^{T} A_{\omega}^{-1} \mathbf{b}_{\omega}+\frac{1}{2} \sqrt{\Delta_{\omega}} \omega^{T} \omega / \lambda_{\omega}
$$

In case (i)

$$
t_{e} \leqslant t_{0}+2 / \sqrt{-\Delta_{\omega}}\left\{\pi / 2-\arctan \left[2 \omega^{T}\left(\mathbf{x}_{0}+\frac{1}{2} A_{\omega}^{-1} \mathbf{b}_{\omega}\right) \lambda_{\omega} / \omega^{T} \omega\right] / \sqrt{-\Delta_{\omega}}\right\} .
$$

In case (ii)

$$
t_{e} \leqslant t_{0}+1 / \sqrt{\Delta_{\omega}} \ln \left[\frac{\omega^{T}\left(\mathbf{x}_{0}+\frac{1}{2} A_{\omega}^{-1} \mathbf{b}_{\omega}\right)+\frac{1}{2} \sqrt{\Delta_{\omega}} \omega^{T} \omega / \lambda_{\omega}}{\omega^{T}\left(\mathbf{x}_{0}+\frac{1}{2} A_{\omega}^{-1} \mathbf{b}_{\omega}\right)-\frac{1}{2} \sqrt{\Delta_{\omega}} \omega^{T} \omega / \lambda_{\omega}}\right] .
$$

Proof. Multiplying the $i$ th equation of (1) by $\omega_{i}, i=1, \ldots, n$ and adding these equations, we have along solutions of (1) that the following equality holds

$$
\frac{d \omega^{T} \mathbf{x}}{d t}=\mathbf{x}^{T} A_{\omega} \mathbf{x}+\mathbf{b}_{\omega}^{T} \mathbf{x}+c_{\omega}
$$

$A_{\omega}$ is assumed positive definite so that we obtain from (2) after completing the square and using the Cauchy-Schwarz inequality, the inequality

$$
\frac{d \omega^{T} \mathbf{x}}{d t} \geqslant\left(\lambda_{\omega} / \omega^{T} \omega\right)\left[\left(\omega^{T} \mathbf{x}\right)^{2}+\omega^{T} A_{\omega}^{-1} \mathbf{b}_{\omega}\left(\omega^{T} \mathbf{x}\right)+\left(\frac{1}{2} \omega^{T} A_{\omega}^{-1} \mathbf{b}_{\omega}\right)^{2}\right]+c_{\omega}-\frac{1}{4} \mathbf{b}_{\omega}^{T} A_{\omega}^{-1} \mathbf{b}_{\omega} .
$$

Since the solution $u\left(t ; u\left(t_{0}\right), t_{0}\right)$ to the scalar equation

where

$$
\frac{d u}{d t}=k_{1} u^{2}+k_{2} u+k_{3} \quad u\left(t_{0}\right)=\omega^{T} \mathbf{x}_{0}
$$

$$
\begin{aligned}
& k_{1}=\lambda_{\omega} / \omega^{T} \omega \\
& k_{2}=\left(\lambda_{w_{1}} / \omega^{T} \omega\right) \omega^{T} A_{. .}^{-1} \mathbf{b}_{a}
\end{aligned}
$$


and

$$
k_{3}=\left(\lambda_{\omega} / \omega^{T} \omega\right)\left(\frac{1}{2} \omega^{T} A_{\omega}^{-1} \mathbf{b}_{\omega}\right)^{2}+c_{\omega}-\frac{1}{4} \mathbf{b}_{\omega}^{T} A_{\omega}^{-1} \mathbf{b}_{\omega}
$$

is a lower bound to $\omega^{T} \mathbf{x}$, we have that $\omega^{T} \mathbf{x}$ escapes in a finite time, i.e. $\left\|\mathbf{x}\left(t ; x_{0}, t_{0}\right)\right\| \rightarrow$ $\infty$, whenever $u\left(t ; \omega^{T} \mathbf{x}_{0}, t_{0}\right)$ does. By finding the roots of the right hand side of (4) and noting that $k_{1}>0$, conditions (i) and (ii) are derived directly. Also since $\mathbf{x}(t$; $\left.\mathbf{x}_{0}, t_{0}\right)$ will escape not later than $u\left(t ; \omega^{T} \mathbf{x}_{0}, t_{0}\right)$ the upper bound for $t_{e}$ is easily computed from the finite escape time for $u\left(t ; \omega^{T} \mathbf{x}_{0}, t_{0}\right)$.

\section{The Matrix Riccati Equation}

In this section we apply Theorem 2.1 to the autonomous matrix Riccati equation

$$
\frac{d P}{d t}=Q+P F+F^{T} P-P R P \quad P\left(t_{0}\right)=\Pi,
$$

where all matrices belong to $\mathbb{R}^{n \times n}$ and $Q, R$ and $\Pi$ and hence $P\left(t ; P_{0}, t_{0}\right)$ are taken to be symmetric. The existence of solutions to (5) on $\left[t_{0}, \infty\right)$ is an important consideration in the Kalman-Bucy filtering theory and the optimal linear-quadratic control problem where equations of type (5) arise. $R$ is usually assumed to be positive definite.

Many sufficient conditions are known for the solution of (5) to exist on $\left[t_{0}, \infty\right)$ (Bucy, 1967; Jacobson, 1970; Rodriguez-Canabal, 1970). In particular, if the Kalman conditions (Jacobson, 1970)

$$
R>0, \quad Q \geqslant 0 \text { and } \Pi \geqslant 0
$$

hold, a solution to (5) exists on $\left[t_{0}, \infty\right)$.

The sufficiency results for the solution of (5) to escape in a finite time, as presented below, thus complement known conditions for solutions of (5) to exist and hence the results can be applied to filtering and regulating problems in optimal control.

The results of the previous section cannot, however, be directly applied to (5) in its matrix form. To convert (5) into a suitable form for analysis the following mathematical devices must be defined and elementary properties used.

(i) The Kronecker product $A \otimes B$ of any two matrices $A \in \mathbb{R}^{m \times n}$ and $B \in \mathbb{R}^{p \times q}$ is defined (Nissan, 1968; Bellman, 1970) as a matrix $C \in \mathbb{R}^{m p \times n q}$ whose elements in the $(i-1) p+1$ to $i p$ rows and $(j-1) q+1$ to $j q$ columns are $\left(a_{i j} B\right), i=1, \ldots, m$, $j=1, \ldots, n$. i.e.

It is easily seen that

$$
C=\left[\begin{array}{c}
a_{11} B \ldots a_{1 n} B \\
\cdot \\
\cdot \\
\cdot \\
a_{m 1} B \\
\ldots
\end{array}\right]
$$

and

$$
(A \otimes B)^{T}=A^{T} \otimes B^{T}
$$

$$
(A \otimes B)^{-1}=A^{-1} \otimes B^{-1} \quad A, B \text { non-singular. }
$$

(ii) For any matrix $A \in \mathbb{R}^{m \times n}$ define a stacking operator $\sigma(A)$ as an $m n$-vector formed by stacking the columns of $A$ under each other, in order, starting with the first; i.e.

$$
\sigma(A)=\left(a_{11}, \ldots, a_{m 1} ; a_{12}, \ldots, a_{m 2} ; \ldots ; a_{1 n}, \ldots, a_{m n}\right)^{T} .
$$


$\sigma$ is easily seen to be a linear operator from $\mathbb{R}^{m \times n}$ to $\mathbb{R}^{m n}$, i.e. for $A, B \in \mathbb{R}^{m \times n}$ and $c \in \mathbb{R}$

$$
\begin{aligned}
\sigma(A+B) & =\sigma(A)+\sigma(B) \\
\sigma(c B) & =c \sigma(B) .
\end{aligned}
$$

(iii) For any matrices $A, B$ and $P$ for which the matrix product $A P B$ is defined, it can be shown that (Nissan, 1968)

$$
\sigma(A P B)=\left(B^{T} \otimes A\right) \sigma(P) .
$$

Using (i)-(iii) above and the symmetry conditions on $P, Q, R$ and $\Pi$ we can prove the following lemma.

LEMMA 3.1. Let $W \in \mathbb{R}^{n \times n}$ be a given constant symmetric matrix. Then along solutions to (5) the following equality is satisfied

$$
\frac{d}{d t} \boldsymbol{\omega}^{T} \mathbf{p}(t)=\mathbf{p}^{T}(t) R_{\omega} \mathbf{p}(t)+\mathbf{f}_{\omega}^{T} \mathbf{p}(t)+\omega^{T} \mathbf{q}
$$

where

and $I$ is the identity matrix.

$$
\begin{aligned}
\omega & =\boldsymbol{\sigma}(W) \\
\mathbf{p} & =\sigma(P) \\
\mathbf{q} & =\sigma(Q) \\
\mathbf{f}_{\omega} & =[F \otimes I+I \otimes F] \boldsymbol{\sigma}(W) \\
R_{\boldsymbol{\omega}} & =-W \otimes R
\end{aligned}
$$

Proof. We have (as in Theorem 2.1) along solutions to (5) that

$$
\frac{d}{d t} \sigma(W)^{T} \sigma(P)=\sigma(W)^{T} \sigma\left(Q+P F+F^{T} P-P R P\right)
$$

Now

Similarly

$$
\begin{aligned}
\sigma(W)^{T} \sigma(P R P) & =\sigma(W)^{T}(I \otimes P R) \sigma(P) \\
& =\sigma(R P W)^{T} \sigma(P) \\
& =\sigma(P)^{T} W \otimes R \sigma(P)
\end{aligned}
$$

$$
\sigma\left(P F+F^{T} P\right)=\left[F^{T} \otimes I+I \otimes F^{T}\right] \sigma(P) .
$$

Using the above, (7) is easily seen to be true.

Comparing equations (7) and (2) we can immediately apply Theorem 2.1 to equation (5) as follows.

THEOREM 3.2. The solution $P\left(t ; \Pi, t_{0}\right)$ of the matrix Riccati equation

$$
\frac{d P}{d t}=Q+P F+F^{T} P-P R P \quad P\left(t_{0}\right)=\Pi
$$

where all matrices belong to $\mathbb{R}^{n \times n}$ and $P, Q, R$ (non-singular) and $\Pi$ are symmetric, has a finite escape time if there exists a symmetric matrix $W \in \mathbb{R}^{n \times n}$ such that $-W \otimes R>0$ and

(i) $\Delta(W, F, R, Q)<0$ or

(ii) $\Delta(W, F, R, Q) \geqslant 0$ and

$$
\begin{aligned}
\sigma(W)^{T} \sigma(\Pi)> & \frac{1}{2} \sigma(W)^{T} W^{-1} \otimes R^{-1}(F \otimes I+I \otimes F) \sigma(W) \\
& +\frac{1}{2} \sqrt{\Delta(W, F, R, Q) \sigma(W)^{T} \sigma(W) / \lambda}
\end{aligned}
$$


where

$$
\begin{aligned}
\Delta(W, F, R, Q)= & -\left[\lambda / \sigma(W)^{T} \sigma(W)\right] \\
& {\left[\sigma(W)^{T}(F \otimes I+I \otimes F)^{T} W^{-1} \otimes R^{-1}(F \otimes I+I \otimes F) \sigma(W)\right.} \\
& \left.+4 \sigma(W)^{T} \sigma(Q)\right]
\end{aligned}
$$

and $\lambda>0$ is the smallest eigenvalue of $-W \otimes R$.

Notes.

(a) The $n^{2}$-vector $\sigma(P)$ actually contains $n(n+1) / 2$ independent elements, since by symmetry considerations $p_{i j}=p_{j i}$ for all $i, j$ provided $i \neq j$.

(b) If $R>0$, which is the case in the standard linear-quadratic optimal control problem, then $-W \otimes R>0$ whenever $-W>0$. In many cases a suitable $W$ may be $W=-I$.

(c) Since properties (i), (ii) and (iii) for Kronecker products as stated above are not exclusive to symmetric or even square matrices, Theorem 2.1 can, using the methods of this section, be applied to more general quadratic matrix equations than (5).

\section{Example 3.3}

The following simple example demonstrates the application of Theorem 3.2 in conjunction with sufficiency results, for example those stated in (6), to an analysis of the existence of solutions of the matrix Riccati equation.

In (5) let $P \in \mathbb{R}^{2 \times 2}, R=2 F=I_{2}$

$$
Q=\left[\begin{array}{ll}
q_{11} & q_{12} \\
q_{21} & q_{22}
\end{array}\right] \text { and } \Pi=\left[\begin{array}{ll}
\pi_{11} & \pi_{12} \\
\pi_{21} & \pi_{22}
\end{array}\right]
$$

where $I_{n}$ will denote the $n \times n$ identity matrix, i.e.

$$
\dot{P}=-P^{2}+P+Q \quad P\left(t_{0}\right)=\Pi \text {. }
$$

From conditions (6) we have that $P\left(t ; \Pi, t_{0}\right)$ exists on $\left[t_{0}, \infty\right)$ provided $Q \geqslant 0$ and $\Pi \geqslant 0$.

Apply Theorem 3.2 by choosing $W=-I_{2}$. Then

$$
\begin{aligned}
-W \otimes R & =I_{4} \Rightarrow \lambda=1 \\
I \otimes F+F \otimes I & =I_{4} \\
\sigma(W)^{T} \sigma(W) & =2 \\
\sigma(W)^{T} \sigma(Q) & =-\left(q_{11}+q_{22}\right)=- \text { trace } Q \\
\sigma(W)^{T} \sigma(\Pi) & =-\left(\pi_{11}+\pi_{22}\right)=- \text { trace } \Pi .
\end{aligned}
$$

Hence

$$
\Delta(W, F, R, Q)=1+2 \text { trace } Q .
$$

Using the results stated in Theorem 3.2 we have that the solution $P\left(t ; \Pi, t_{0}\right)$ to (8) escapes in a finite time:

(i) for all $\Pi$, whenever trace $Q<-\frac{1}{2}$;

(ii) for trace $\Pi<1-\sqrt{1+2 \text { trace } Q}$ whenever trace $Q \geqslant-\frac{1}{2}$.

We have, from the above analysis, that

(a) when $Q=0, P\left(t ; \Pi, t_{0}\right)$ exists on $\left[t_{0}, \infty\right)$ whenever $\Pi \geqslant 0$, and escapes in a finite time whenever trace $\Pi<0$;

(b) when $\Pi=0, P\left(t ; \Pi, t_{0}\right)$ exists on $\left[t_{0}, \infty\right)$ whenever $Q \geqslant 0$, and escapes in a finite time whenever $\sqrt{2 \text { trace } Q+1}-1<0$ or trace $Q<-\frac{1}{2}$, i.e. trace $Q<0$. 
Since the stipulation that the trace of a matrix should have a definite sign is much less restrictive than requiring the matrix itself to be definite, the finite escape time conditions in (a) and (b) above are less restrictive in form than the Kalman existence conditions.

However, in (ii) above we see that the condition on trace $\Pi$ is dependent on trace $Q$ so that we cannot state conditions on $\Pi$ and $Q$ independently of each other as we can for the Kalman existence conditions applied to equation (8). This restriction in stating sufficiency results for a finite escape time to exist can be minimized by selecting a $W$ (other than $I$ ) which will (in some sense) maximize the range of $Q$ 's and $\Pi$ 's which will satisfy the inequality given in (ii) above.

\section{Models in Population Dynamics}

A mathematical model frequently used to describe the dynamic behaviour of $n$ interacting populations $x_{1}, x_{2}, \ldots, x_{n}$ is given by a system of quadratic differential equations

$$
\dot{x}_{i}=x_{i}\left(\sum_{j=1}^{n} a_{i j} x_{j}+\varepsilon_{i}\right) \quad x_{i}\left(t_{0}\right)=x_{i 0}>0 \quad i=1, \ldots, n .
$$

Equation (9) can be written in the format of equation (2) as follows.

Let

Hence

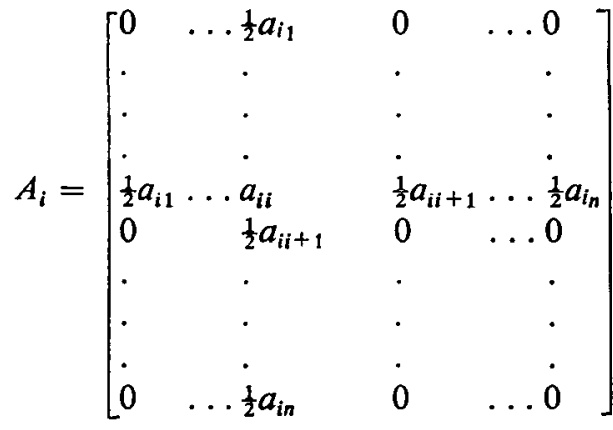

$$
\begin{aligned}
& \mathbf{b}_{i}^{T}=\left(0, \ldots, 0, \varepsilon_{i}, 0, \ldots, 0\right) \\
& c_{\mathrm{t}}=0 \text {. }
\end{aligned}
$$

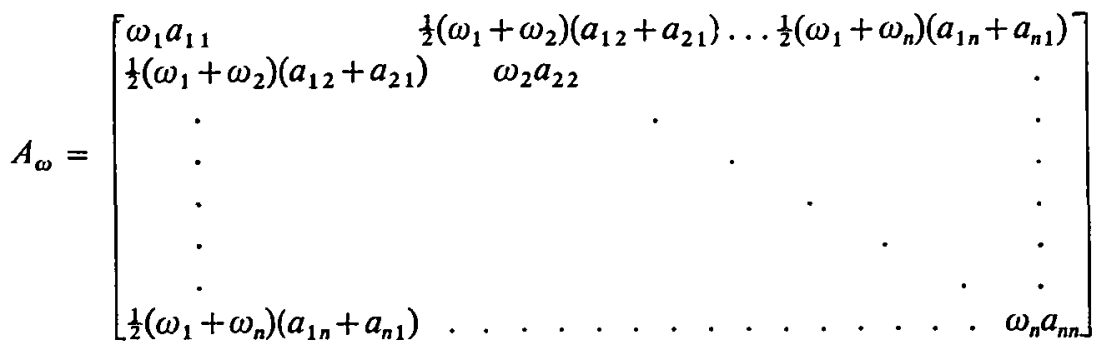

$$
\begin{aligned}
& \mathbf{b}_{\omega}^{T}=\left(\omega_{1} \varepsilon_{1}, \omega_{2} \varepsilon_{2}, \ldots, \omega_{n} \varepsilon_{n}\right) \\
& c_{\omega}=0 \text {. }
\end{aligned}
$$

Since $c_{\omega}=0$, condition (ii) of Theorem 2.1 must always hold under the assumption that $A_{\omega}>0$. Then the condition on $\omega^{T} \mathrm{x}_{0}$ becomes

$$
\omega^{T} \mathbf{x}_{0}>-\frac{1}{2} \omega^{T} A_{\omega}^{-1} \mathbf{b}_{\omega}+\frac{1}{2} \sqrt{\left(\omega^{T} \omega\right) \mathbf{b}_{\omega}^{T} A_{\omega}^{-1} \mathbf{b}_{\omega} / \lambda_{\omega}}
$$


Since $A_{\omega}$ is symmetric and positive definite and $\lambda_{\omega}>0$ is its smallest eigenvalue, we have that $1 / \lambda_{\omega}>0$ is the largest eigenvalue of $A_{\omega}^{-1}$. Hence it is easily seen that

using the Cauchy-Schwarz inequality.

$$
\begin{aligned}
\left(\omega^{T} \omega\right) \mathbf{b}_{\omega}^{T}\left(A_{\omega}^{-1} / \lambda_{\omega}\right) \mathbf{b}_{\omega} & \geqslant\left(\omega^{T} \omega\right) \mathbf{b}_{\omega}^{T} A_{\omega}^{-1} A_{\omega}^{-1} \mathbf{b}_{\omega} \\
& \geqslant\left(\omega^{T} A_{\omega}^{-1} \mathbf{b}_{\omega}\right)^{2}
\end{aligned}
$$

Hence

and thus

$$
\pm \sqrt{\left(\omega^{T} \omega\right) \mathbf{b}_{\omega}^{T} A_{\omega}^{-1}} \overline{\mathbf{b}_{\omega} / \lambda_{\omega}} \geqslant \omega^{T} A_{\omega}^{-1} \mathbf{b}_{\omega}
$$

$$
\omega^{T} \mathbf{x}_{0}>-\frac{1}{2} \omega^{T} A_{\omega}^{-1} \mathbf{b}_{\omega}+\frac{1}{2} \sqrt{\left(\omega^{T} \omega\right) \mathbf{b}_{\omega}^{T} A_{\omega}^{-1} \mathbf{b}_{\omega} / \lambda_{\omega}} \geqslant 0
$$

Note that this analysis applies in general to the condition on $\omega^{T} \mathbf{x}_{0}$ in case (ii) of Theorem 3.2, whenever $c_{\omega}=0$.

Using the above forms for $A_{\omega}$ and $\mathbf{b}_{\omega}$ we can apply Theorem 2.1 to equation (9) to derive a set of sufficient conditions for the solution to (9) to exhibit a finite escape time.

\section{Conclusion}

In this paper we have presented sufficiency conditions for solutions to systems of autonomous quadratic differential equations to exhibit a finite escape time. Trivially, these conditions can be applied to non-autonomous non-linear systems whose solutions are bounded below in some suitable manner by solutions to autonomous quadratic differential equations.

A restriction on the application of the results presented in this paper is the requirement that there exists an $\omega \in \mathbb{R}^{n}$ such that $\sum_{i} \omega_{i} A_{i}>0$.

\section{REFERENCES}

BellmaN, R. E. 1970 Introduction to matrix analysis, 2nd edn. New York: McGraw-Hill. BucY, R. S. 1967 J. Comput. Syst. Sci. 1, 349-361.

CESARI, L. 1963 Asymptotic behaviour and stability problems in ordinary differential equations, 2nd edn. Berlin, Göttingen, Heidelberg: Springer-Verlag.

HAHN, W. 1967 Stability of motion. Berlin, Heidelberg, New York: Springer-Verlag.

JACOBSon, D. H. 1970 J. Diff. Equ. 8, 258-263.

NisSAN, D. H. 1968 Econometrica 36, 603-604.

Rodriguez-Canabal, J. 1973 Stochastics 1, 129-149.

WilLEMS, J. L. 1970 Stability theory of dynamical systems. London: Thomas Nelson \& Sons Ltd. 